\title{
Caso raro de urolitíase de fosfato de cálcio em felino com dois meses de idade
}

\author{
Rare case of calcium phosphate urolithiasis in 2-month-old feline \\ Caso raro de urolitiasis de fosfato de cálcio en felino con dos meses de edad
}

\author{
Eduarda Santos Bierhals \\ ORCID: https://orcid.org/0000-0002-9604-8580 \\ Universidade Federal de Pelotas, Brasil \\ E-mail: dudabierhals@hotmail.com \\ Andreza Bernardi da Silva \\ ORCID: https://orcid.org/0000-0002-9740-5308 \\ Universidade Federal de Pelotas, Brasil \\ E-mail: bernardiandreza@gmail.com \\ Alan Carlos de Santana \\ ORCID: https://orcid.org/0000-0002-6673-9700 \\ Universidade Federal de Pelotas, Brasil \\ E-mail: alan.carlos1983@yahoo.com.br \\ Filipe Costa da Silva \\ ORCID: https://orcid.org/0000-0002-9604-8580 \\ Universidade Federal de Pelotas, Brasil \\ E-mail: lipe.spts@gmail.com \\ Péter de Lima Wachholz \\ ORCID: https://orcid.org/0000-0002-7616-6766 \\ Universidade Federal de Pelotas, Brasil \\ E-mail: peterlwachholz@gmail.com \\ Vitória Ramos de Freitas \\ ORCID: https://orcid.org/0000-0001-6725-6789 \\ Universidade Federal de Pelotas, Brasil \\ E-mail: vitoriabars@hotmail.com \\ Thaís Cozza dos Santos \\ ORCID: https://orcid.org/0000-0002-2049-1463 \\ Universidade Federal de Pelotas, Brasil \\ E-mail: thcs@live.com \\ Pâmela Caye \\ ORCID: https://orcid.org/0000-0002-6456-8100 \\ Universidade Federal de Santa Maria, Brasil \\ E-mail: pamiscaye@gmail.com \\ Alexsander Ferraz \\ ORCID: https://orcid.org/0000-0002-0424-6249 \\ Universidade Federal de Pelotas, Brasil \\ E-mail: xanderferraz@yahoo.com.br \\ Márcia de Oliveira Nobre \\ ORCID: https://orcid.org/0000-0003-3284-9167 \\ Universidade Federal de Pelotas, Brasil \\ E-mail: marciaonobre@gmail.com
}

\begin{abstract}
Resumo
A urolitíase é uma das principais doenças relacionadas ao trato urinário inferior de felinos. O objetivo deste trabalho foi relatar um raro caso de paciente felino pediátrico diagnosticado com urolitíase de fosfato de cálcio aos dois meses de idade. Dessa forma, foi realizada ultrassonografia abdominal completa onde foi constatada a presença de uma estrutura em região caudal da vesícula urinária, ocupando $80 \%$ do tamanho total vesical e a presença de urina era ínfima, impossibilitando a realização de urinálise. Assim, a paciente foi encaminhada para realização de cistotomia para remoção da urolitíase. Durante o procedimento cirúrgico foi observada a presença de diversos micro urólitos, os quais estavam agrupados formando um único objeto de formato irregular e coloração amarelada. Através de análise mineralógica foi detectada presença de $100 \%$ de fosfato de cálcio hidroxilado, traços de oxalato de cálcio monohidratado e traços de matriz orgânica amorfa. A presença de urólitos de fosfato de cálcio em felinos adultos, já é pouco frequente, quando se trata de filhotes não há registro na literatura. Ou seja, este caso se demonstra raro pela idade da paciente atendida e pela composição da urolitíase diagnosticada em sua vesícula urinária.
\end{abstract}

Palavras-chave: Trato urinário inferior; Urólitos; Disúria; Felino pediátrico. 


\begin{abstract}
Urolithiasis is one of the most important diseases related and relevant to the lower urinary tract of cats. This study aimed to report a rare case of a pediatric feline patient diagnosed with calcium phosphate urolithiasis. Thus, a complete abdominal ultrasound was performed, which revealed the presence of a structure in the caudal region of the urinary bladder, occupying virtually $80 \%$ of the total bladder size, there was a small quantity of urine present, so collection for urinalysis. Consequently, the patient was referred for cystotomy for the removal of urolithiasis. During the surgical procedure, several microuroliths were observed, which were grouped together, forming a single object of irregular shape and yellowish color. Through mineralogical analysis was detected the presence of $100 \%$ hydroxylated calcium phosphate, traces of calcium oxalate monohydrate, and traces of amorphous organic matrix. The presence and total composition of calcium phosphate in feline uroliths is much unusual in adults, and to the Best of our knowledge there are no records of it in kittens. This disease in a pediatric patient has never been known to the best of our knowledge. So, this case is rare one for the age of the patient and the composition of the urolithiasis diagnosed in her urinary bladder and because of her early age.
\end{abstract}

Keywords: Lower urinary tract; Uroliths; Disury; Pediatric cats.

\title{
Resumen
}

La urolitiasis es una de las principales enfermedades relacionadas con el tracto urinario inferior de los felinos. El objetivo de este trabajo es relatar un raro caso de un paciente felino pediátrico diagnosticado con urolitiasis de fosfato cálcico a los dos meses de edad. De esta forma, fue realizada ecografía abdominal en donde fue constatada la presencia de una estructura en la región caudal de la vejiga, ocupando 80\% del tamaño vesical, imposibilitando la realización de uroanálisis debido a la pequeña cantidad de orina. Posteriormente, la paciente fue conducida al quirófano para realizar cistotomía para remoción de la urolitiasis. Durante el procedimiento quirúrgico fue observado la presencia de diversos micro urolitos, los cuales estaban agrupados formando una única estructura de formato irregular y coloración amarilla. En el análisis mineral fue detectada presencia de $100 \%$ de fosfato cálcico hidroxilado, trazos de oxalato cálcico monohidratado y trazos de matriz orgánica amorfa. La presencia de urolitos de fosfato de calcio en felinos adultos, es poco frecuente, cuando se trata de gatitos no hay registro en la literatura. Por tanto, este caso se demuestra por la edad de la paciente atendida y por la composición del urolito diagnosticado en vejiga urinaria.

Palabras clave: Tracto urinario; Urolitos; Disuria; Felino pediátrico.

\section{Introdução}

Dentre as diversas enfermidades que acometem a espécie felina, salienta-se as relacionadas ao trato urinário inferior (TUI), sendo a urolitíase uma das principais afecções deste sistema, não apenas por sua alta incidência, mas também pela relevância clínica na medicina felina (Rick et al., 2017). A urolitíase é complexa e multifatorial, devido ao fato de que diversos cristaloides podem estar envolvidos em sua formação.

A prevalência dos urólitos na espécie felina se dá majoritariamente por fosfato triplo (estruvita) e oxalato de cálcio. Ainda assim, outras composições podem precipitar formando cálculos, e por mais que pouco frequentes, são observados também minerais como urato, fosfato de cálcio, cistina e sílica (Gomes et al., 2018). Quanto aos sinais clínicos, eles dependem da localização, tamanho e quantidade de urólitos presentes, não havendo sinais específicos, o que acarreta com que não se consiga diferenciar clinicamente de outras enfermidades no TUI (Houston et al., 2016). Alguns dos fatores de risco estão relacionados à raça, sexo, idade, anormalidades anatômicas e funcionais do trato urinário, anormalidades de metabolismo, infecções do trato urinário, dieta, pH urinário e homeostase hídrica corporal (Bartges, 2016). Dentre as raças mais acometidas, encontram-se Himalaia, Persa e Gato Americano de Pelo Curto (Stevenson, 2002). Gatos das raças Cornish Rex, Burmês, Azul Russo, Sagrado da Birmânia e Siamês tem menor probabilidade de desenvolverem urolítiases a base de fosfato triplo (Lekcharoensuk, et al. 2001; Little, 2016).

Dessa forma, o presente trabalho teve como objetivo apresentar um estudo de caso descritivo (Yin, 2015) por se tratar de um raro caso de urolitíase a base de fosfato de cálcio em paciente felino com apenas dois meses de idade.

\section{Metodologia e Relato de Caso}

Um felino, fêmea, sem raça definida (SRD), com 2 meses de idade, pesando 840 gramas, foi atendido pelo setor de 
Clínica Médica de Animais de Companhia do Hospital de Clínicas Veterinárias da Universidade Federal de Pelotas (HCV/UFPel). De acordo com o relatado pela tutora durante o atendimento, o felino há cerca de quatro dias apresentava manifestações clínicas como, disúria, polaciúria, hematúria e agressividade. A paciente havia sido adotada há apenas uma semana. Conforme anamnese, a dieta do felino era baseada em alimento seco comercial standard específico para filhotes. Durante o exame clínico, foi observado escore corporal ideal, mucosas normocoradas, normohidratação, linfonodos fisiológicos e temperatura retal de $37,8^{\circ} \mathrm{C}$. Na palpação abdominal constatou-se rigidez abdominal com presença de desconforto e dor ao toque, sendo esta a única alteração no exame físico específico. Foi observada a presença de pequenas gotas de urina com coloração avermelhada ao deixar a paciente sobre a mesa de atendimento. A partir disto, foi solicitada a realização de exames complementares, como hemograma completo, teste de FIV/FeLV e ultrassonografia abdominal completa no Laboratório de Diagnóstico por Imagem e Cardiologia da UFPel (LADIC/UFPel). Devido ao comportamento agressivo da felina a mesma foi submetida à sedação, com administração de dexmedetomidina $40 \mathrm{mcg} / \mathrm{kg}$ via intramuscular, para realização de detalhado exame clínico, coleta de amostras biológicas para exames hematológicos e sorológicos e ainda exames de imagem tendo sido realizada também tentativa de colheita urinária através de cistocentese guiada por ultrassom para urinálise.

O resultado do teste de FIV/FeLV foi negativo para ambas as retroviroses. Quanto ao hemograma, foi constatado início de liberação de bastonetes 171 microlitros $(\mu \mathrm{L})(0$ - 300 $\mu \mathrm{L})$, porém sem leucocitose completa e, ainda, linfocitose $11.457 \mu \mathrm{L}(1.500-7.000 \mu \mathrm{L})$. Durante a realização do exame de ultrassonografia abdominal, foi observado formato anatômico e dimensões preservadas dos órgãos da cavidade abdominal. Porém, na vesícula urinária, foi notada ausência de repleção de conteúdo e visualizada a presença de estrutura em região caudal vesical com contornos indefinidos e hiperecogênicos com parênquima heterogêneo, sendo este hipoecoico e anecoico com sinal de doppler negativo, o que sugeria a presença de urolitíase vesical (Carvalho, 2014). Tal estrutura ocupava cerca de $80 \%$ do diâmetro da vesícula urinária e média aproximadamente $0,71 \times 0,58 \times 0,55$ centímetros $(\mathrm{cm})$ (Figura 1 e 2 ).

Foi realizada tentativa de cistocentese para realização de urinálise, todavia, devido à fração ínfima de urina na bexiga em função da polaciúria apresentada pela paciente e o grande volume do urólito que ocupava cerca de $80 \%$ da bexiga não foi viável coleta de quantidade suficiente para esta análise.

Figura 1: Ecografia abdominal de felino, fêmea, 2 meses de idade, apresentando sinais clínicos de alteração em TUI, imagem de corte transversal da vesícula urinária, evidenciando a presença de estrutura compatível com urolitíase.

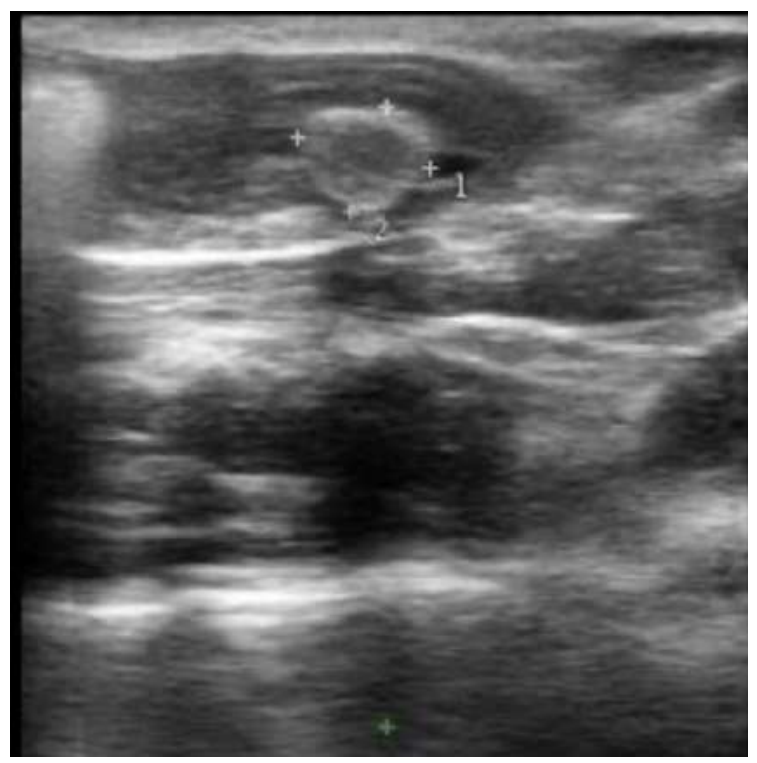

Fonte: LADIC/UFPel. 
Figura 2: ecografia abdominal de felino, fêmea, 2 meses de idade, apresentando sinais clínicos de alteração em TUI, imagem de corte longitudinal da vesícula urinária, evidenciando a presença de estrutura hiperecogênica com parênquima heterogêneo, compatível com urolitíase.

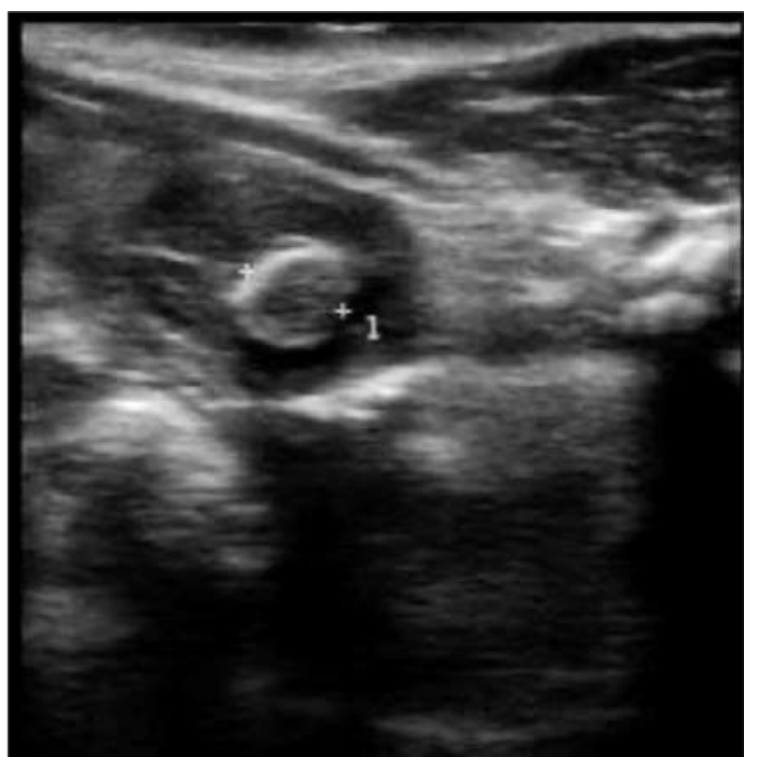

Fonte: LADIC/UFPel.

Devido aos resultados da ultrassonografia abdominal e exame clínico a paciente foi encaminhada para realização de cistotomia. Este procedimento cirúrgico teve início com realização de incisão retroumbilical mediana, após foi feita cistocentese e posicionamento de pontos de reparo para realização de incisão de $3 \mathrm{~cm}$ dorsais à bexiga. Foram removidos os urólitos e realizada lavagem abundante com solução fisiológica de cloreto de sódio a $0,9 \%$. Foi posicionada sonda uretral $\mathrm{N}^{\mathrm{o}} 4$ em direção normógrada, realizada sutura vesical e feito teste de extravasamento que foi negativo. Dessa forma, prosseguiu-se para realização de celiorrafia e fixação de sonda uretral, finalizando o procedimento. Durante a cirurgia foi observada a presença de diversos micro urólitos, os quais estavam agrupados formando um único objeto de formato irregular e coloração amarelada. Estes foram removidos e armazenados para posterior envio para análise mineralógica.

Os cuidados pós-cirúrgicos consistiram em realização de limpeza de ferida cirúrgica a cada 12 horas, tal procedimento recomendado até a remoção de pontos em 10 dias após a cirurgia, analgesia com administração de dipirona $20 \mathrm{mg} / \mathrm{kg}$ a cada 12 horas durante 7 dias, administração de tramadol 3mg/kg a cada 12 horas durante 3 dias e meloxicam $0,05 \mathrm{mg} / \mathrm{kg}$ a cada 24 horas durante 3 dias. Além disso, enquanto a paciente permaneceu internada no hospital teve seus parâmetros clínicos avaliados três vezes ao dia, e recebeu como dieta alimentação úmida para filhotes e água à vontade. A sonda uretral foi removida 48 horas após o procedimento. A felina permaneceu internada por 3 dias, e ao se verificar a presença de urina com coloração fisiológica e micção em quantidade normal, recebeu alta e foi levada de volta ao seu domicílio.

O urólito foi enviado ao Litolab, laboratório de mineralogia de cálculos urinários, em Porto Alegre/RS, para análise de sua composição química e dimensões, o qual se apresentou como $100 \%$ fosfato de cálcio hidroxilado, traços de oxalato de cálcio monohidratado e traços de matriz orgânica amorfa (Figura 3) de tamanho 2,0 x 2,0 x 4,5 milímetros pesando 0,007 gramas. 
Figura 3: Imagem de urolitíase de paciente felino, fêmea, com 2 meses de idade, fornecido através do laudo elaborado pelo Litolab (Laboratório de Mineralogia de Cálculos Urinários - Porto Alegre/RS) - Largura dos retângulos = 1mm.

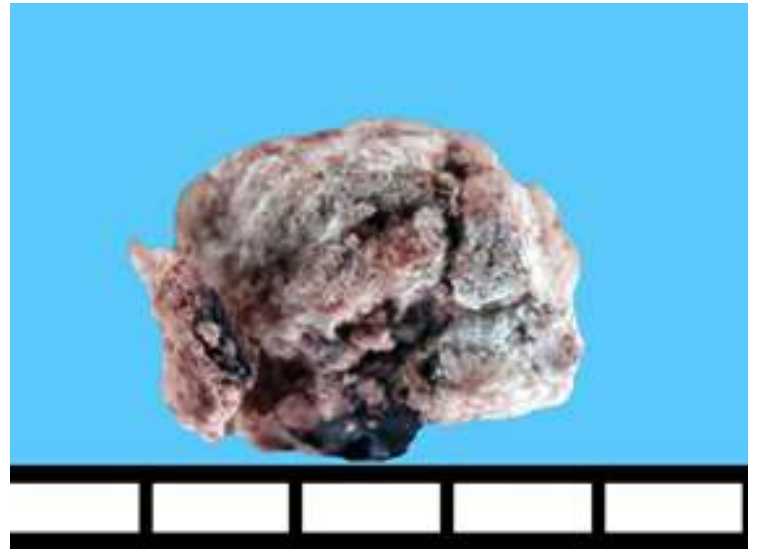

Fonte: Litolab.

No $10^{\circ}$ dia após a cirurgia a paciente retornou para remoção de pontos e foi relatado pela tutora que a paciente se apresentava estável e em condições fisiológicas: sem manifestação de dor abdominal, urinando em quantidades e colorações consideradas ideais, alterando também o comportamento agressivo que apresentou nos dias antecedentes da consulta médica para comportamento dócil. Importante salientar que conforme a AAFP (American Association of Feline Practitioners) a agressividade apresentada por felinos pode estar correlacionada a dor preexistente ocasionada por alguma condição médica, e tal alteração foi apresentada pelo felino pediátrico em questão e sanada brevemente após a resolução clínica do caso. Após 30 dias foi repetida ecografia abdominal, onde a vesícula urinária apresentou-se com paredes finas e com aspecto ultrassonográfico fisiológico, e presença de sedimento e conteúdo anecogênico, tal alteração esperada em função da manipulação vesical recente.

No período de 1 ano após a cistotomia, foram realizadas ultrassonografias e urinálises trimestrais para controle de novas formações de urólitos, e durante este período não foram observados indícios de recidivas. Em urinálises não foram visualizados cristais, houve pequena presença de leucócitos (1 por campo) na terceira análise, onze meses após o procedimento cirúrgico, o pH se manteve entre 6,2 e 6,5 e a densidade entre 1.022 e 1.040, sem mais alterações dignas de nota. A vesícula urinária se manteve com paredes finas e não houve presença de sedimento visível ao exame ultrassonográfico (Figura 4). 
Figura 4: Imagem ultrassonográfica da vesícula urinária da paciente felina deste relato de caso após 1 ano do diagnóstico de urolitíase de fosfato de cálcio, agora sem alterações apresentando paredes normoespessas sem presença de estruturas hiperecogênicas.

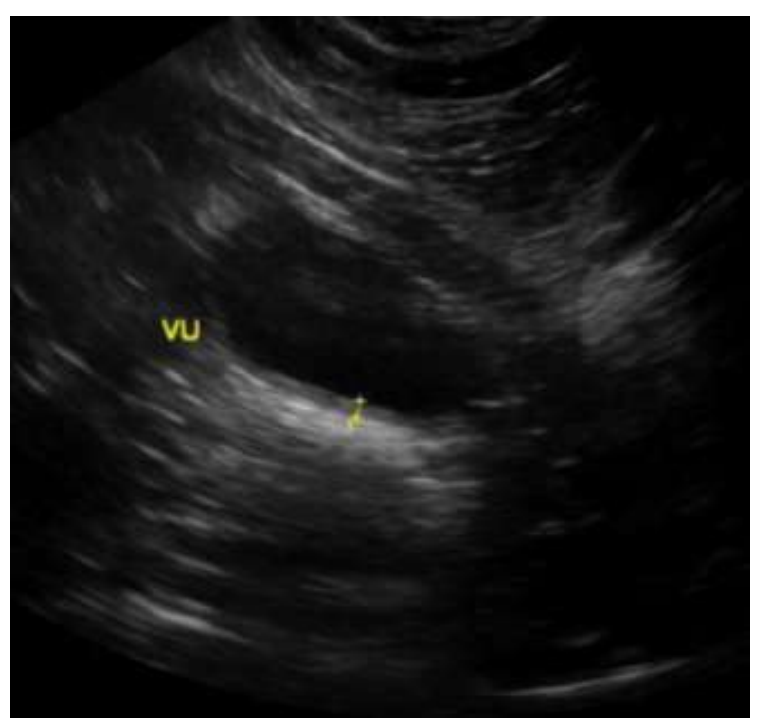

Fonte: LADIC/UFPel.

Em função de se tratar de um paciente pediátrico, não foi possível prescrever dieta calculolítica ou preventiva de urolitíase para esta paciente. A única recomendação além dos exames complementares de acompanhamento trimestral foi o estímulo à ingestão hídrica através de alimentação úmida, fontes de água, distribuição de diversas vasilhas de água em sua residência para a paciente. Com estas recomendações foi possível prevenir uma nova formação de urolitíase.

\section{Resultados e Discussão}

A presença de urólitos de fosfato de cálcio em felinos adultos é pouco frequente, porém quando se trata de filhotes não há registros na literatura consultada. Little (2016) afirma que urólitos a base de estruvita podem ocorrer por infecção no TUI em felinos abaixo de um ano de idade, porém não menciona idade de maior prevalência para ocorrência de formação de urólitos com a composição de fosfato de cálcio. A prevalência de urolitíase felina relatada pela literatura com relação à idade indica que apesar de esta enfermidade poder acometer felinos de toda e qualquer faixa etária, há uma maior frequência em pacientes felinos acima de 8 anos. Independentemente do tipo de urólito encontrado, as idades com maior predisposição e ocorrência de casos não condizem com a presenciada no atual relato, onde o paciente felino apresentava urolitíase de fosfato de cálcio com 2 meses de idade. Felinos adultos jovens, em torno de 1 a 6 anos, tendem a desenvolver urolitíase por fosfato triplo, enquanto felinos com idade superior, de 7 a 10 anos, tendem a ser mais predispostos a formar cálculos de oxalato de cálcio (Mendoza-López et al., 2019). O paciente deste relato apresentava urolitíase de fosfato de cálcio e o urólito principal apresentava tamanho de 2,0 × 2,0 × 4,5 mm, pesando 0,007 gramas.

Não se tem referências sobre a prevalência quanto à raça, como por exemplo, no caso de urólitos de oxalato de cálcio que apresentam uma predisposição maior em felinos de pelos longos. Todavia, com relação ao sexo, fêmeas correspondem à maioria dos casos sendo 57\% destes (Gomes et al., 2018), dado que condiz com este relato. O paciente apresentou polaciúria, disúria e hematúria, e em exame de imagem foi possível visualizar alteração em parede vesical, onde se apresentava espessada no exame pré-cirúrgico, fato que não se repetiu nos exames pós-cirúrgicos, onde sempre foi visualizada a vesícula urinária em seu formato de pêra fisiológico repleta por conteúdo anecogênico e sem sinais de litíases bem como sinais compatíveis com cistite. Isso foi de encontro com o relatado por Del-Angel-Caraza et al. (2020), que relatou que localização, tamanho e 
quantidade de urólitos são características que corroboram para variação dos sinais clínicos da urolitíase, no entanto, sabe-se que os sinais clínicos mais frequentemente associados são polaciúria, disúria e hematúria, alterações compatíveis também com cistite isoladamente ou causada pela presença de urólitos.

De modo geral, o TUI é um sistema perito no serviço de captar, armazenar e liberar a urina concentrada de forma cíclica, eliminando assim possíveis resíduos corporais na forma líquida (Waki \& Kogika, 2015). Esses resíduos, em especial minerais, quando não eliminados de forma correta acarretam supersaturação urinária, podendo ocasionar na precipitação e formação de cristais (Assis \& Taffarel, 2018). Tais cristais quando retidos no sistema urinário podem agregar-se com outros minerais ou matriz orgânica e agrupar-se em concreções sólidas, conhecidas como cálculos urinários ou urólitos (Silva et al., 2020), ou seja, urinas hipersaturadas tem maior presença de sais dissolvidos que predispõem à formação de urolitíase (Grauer, 2015; Grauer, 2005). A presença minerais em determinadas situações pode liberar cristais que podem ser visualizados facilmente em exames qualitativos de urina, entretanto não foi possível realizar urinálise do paciente felino em questão para avaliar este ponto antes da realização de cistotomia. Nas urinálises pós-cirúrgicas não foram visualizados cristais urinários.

Com o decorrer dos anos houve uma grande variação de relatos sobre a prevalência dos urólitos felinos compostos por estruvita (fosfato amoníaco magnesiano hexahidratado ou fosfato triplo) e oxalato de cálcio na rotina de atendimentos das clínicas veterinárias. Em 2006, os urólitos de estruvita representaram 50\% e os de oxalato de cálcio 39\% nas avaliações realizadas, no entanto ocorreu uma alternância em 2019, onde 54\% dos urólitos relatados foram compostos de oxalato de cálcio e apenas 32\% compostos por estruvita. De maneira mais infrequente encontrou-se os demais constituintes de cristais, como sílica, urato, cistina e fosfato de cálcio (Mendoza-López et al., 2019). Ressaltando o componente atípico presente no urólito do relato em questão, o fosfato de cálcio, o qual em diferentes estudos apresentou uma prevalência menor que $1 \%$ (Gomes et al., 2018). O fosfato de cálcio é geralmente visto em associação com outros tipos de minerais, como oxalato de cálcio e estruvita, apenas representando uma pequena fração na composição do urólito, entretanto, foi observado na análise da mineralogia do cálculo do exposto deste relato a composição de 100\% de fosfato de cálcio (Houston et al., 2016). Além disso, é encontrado normalmente em urinas com pH alcalino a neutro, os cristais têm aparência amorfa ou primas longos e esguios além de que a urocultura se apresenta negativa (Lulich et al., 2011).

Não foi possível determinar um motivo para a paciente relatada ter apresentado tal composição de urolitíase em função de sua baixa idade e falta de histórico familiar por ter sido adotada sem procedência. No geral, relaciona-se a formação de tal urolitíase de fosfato de cálcio pela ingestão de fontes extremamente ricas em cálcio e fósforo concomitantemente com a baixa disponibilidade e ingestão hídrica do paciente. Embora, pela carência de pesquisas e relatos relacionados a este componente em decorrência de sua baixa prevalência, não se tem base considerável para discussão quanto aos seus fatores predisponentes, diferente do apresentado por outros compostos como oxalato de cálcio e fosfato triplo. No entanto, potenciais fatores de risco podem incluir hipercalciúria devido à hipercalcemia, desordens hipercalcêmicas como neoplasias, excesso de vitamina $\mathrm{D}$ sérica e cálcio em excesso na dieta do felino, hiperfosfatúria devida ao consumo excessivo de fósforo, além do $\mathrm{pH}$ urinário alcalino e diminuição de volume urinário por polaciúria, a qual pode favorecer a formação de coágulos sanguíneos que podem acarretar hematúria (Gomes et al., 2018). Os fatores de risco para o desenvolvimento de urolitíase são vários e, por esse motivo, o tratamento e a prevenção dessa enfermidade ainda são um desafio mesmo com sua alta ocorrência na clínica médica veterinária de felinos.

Segundo Silva (2020), o diagnóstico da urolitíase é baseado nos sinais clínicos apresentados, no hemograma para detecção de evidências de resposta inflamatória sistêmica, nos exames de imagem como radiografia simples, radiografia contrastada e ultrassonografia, na bioquímica sanguínea que pode se demonstrar necessária para a diferenciação dos cálculos de outras causas com semelhante sintomatologia (afecção idiopática, infecções do TUI e neoplasias), assim como a realização da análise dos urólitos para determinar a composição do mineral em questão. O diagnóstico do paciente relatado quanto aos 
achados ultrassonográficos foi característico de urolitíase, bem como a sintomatologia apresentada por ele. Porém, a urinálise não foi passível de realização devido ao grande volume vesical ocupado pelo cálculo e assim não haver urina presente na vesícula urinária, impossibilitando a coleta.

Através da análise da composição mineral do urólito se obteve o diagnóstico definitivo da urolítiase sendo determinada a composição de $100 \%$ de fosfato de cálcio hidroxilado, com traços oxalato de cálcio monohidratado e traços matriz orgânica amorfa. Salienta-se que para o diagnóstico definitivo dos urólitos é sempre necessária a realização de análise da composição mineral do cálculo, assim as análises quantitativas se tornam de extrema importância, dependendo das mesmas para uma correta classificação e identificação das camadas dos cristais, desse modo os protocolos de tratamento e prevenção devem ser baseados em tais análises (Del-Angel-Caraza et al., 2020).

De acordo com Del-Angel-Caraza et al. (2020), um dos principais pontos de tratamento para tal enfermidade é a dissolução com auxílio de rações medicamentosas ou fármacos alcalinizantes/acidificantes ou remoção cirúrgica do urólito. Em caso de cálculos ativos como os com presença de cálcio em que não podem ser dissolvidos, o tratamento de escolha é a remoção cirúrgica, com a abertura da bexiga para retirada do material para análise, no caso em questão o urólito encontrava-se na vesícula urinária denominando o procedimento cirúrgico como cistotomia. Essa técnica foi primordial para o alívio do paciente o qual apresentava sinais clínicos intensos e elevado desconforto, além de facilitar o manejo e os cuidados necessários do filhote pelo tutor.

A terapêutica instaurada para esse paciente, cistotomia e monitoramento, foi suficiente e após um ano o felino não apresentou mais nenhum indício de recidivas para a afecção sofrida anteriormente, mesmo sem ter havido possibilidade de se intervir com dieta específica para prevenção de urolitíase a base de cálcio. Quanto à prevenção para urolitíases, tem-se recomendação de dietas equilibradas cuidando o teor de umidade com remoção de fontes de alta disponibilidade de minerais como cálcio e fósforo, da mesma forma que um tratamento apropriado e incentivo à ingestão hídrica, importante para manutenção de pH urinário (Rick et al., 2017). Também, é importante promover a diurese através de uma maior ingestão hídrica, erradicar infecções de TUI e aumentar a solubilidade urinária reduzindo a concentração de cristaloides (Ettinger et al., 2017).

Dessa forma, o prognóstico para pacientes com tal patologia é geralmente favorável, sempre tendo em vista ser uma enfermidade comum de apresentar recidivas, mas com o conhecimento da composição do urólito predominante, bem como a eficiência do tratamento prescrito, as recidivas tendem a ser menos frequentes (Assis \& Taffarel, 2018). Uma vez formado, o urólito de fosfato de cálcio tem sua dissolução inviável através de dietas calculolíticas devido ao fato de que mesmo que identificada a fonte principal da hipercalcemia no organismo a sua reversão leva tempo, além de não interferir no cálculo já formado (Ázira, 2016). E mesmo após a resolução atual de um caso de urolitíase de qualquer composição, é importante realizar o acompanhamento dos animais considerados predispostos para que em caso de recidiva futura se faça diagnóstico precocemente, se tenha bom prognóstico e se mantenha uma boa qualidade de vida (Barwaldt et al., 2021), além de que as urolitíases não devem ser classificadas como afecções pontuais, mas como uma consequência de múltiplas alterações sistêmicas em interação (Petricione, 2021).

Este relato é de extrema importância pelo fato de não se ter encontrado na literatura consultada qualquer caso semelhante. Assim determinando a necessidade deste registro tanto para auxiliar aos clínicos veterinários na rotina de atendimento a felinos com distúrbios urinários, quanto na realização de pesquisas e estudos mais específicos sobre o desenvolvimento de urólitos de oxalato de cálcio de grandes dimensões em filhote da espécie felina para se esclarecer possíveis fatores predisponentes, desencadeantes e medidas preventivas destes. 


\section{Considerações Finais}

Com o presente trabalho, relata-se pioneiramente o caso de um felino, fêmea, SRD, diagnosticada com urolitíase de fosfato de cálcio com apenas dois meses de idade. Tal fato ainda não foi relatado anteriormente na literatura consultada, possivelmente em função da baixa frequência de relatos de urolitíases de fosfato de cálcio e de urolitíases de qualquer natureza em felinos com idade inferior a 3 meses de idade. Dessa forma, deve-se incentivar, valorizar e realizar mais pesquisas acerca de urolitíases, suas composições e condições médicas concomitantes para que se consiga determinar futuramente quais são os fatores predisponentes nestes casos e de que forma seria possível prevenir sua ocorrência.

\section{Referências}

AAFP. (2004). Feline Behavior Guidelines. American Association of Feline Practitioners, 6-43.

Assis, M. \& Taffarel, M. (2018). Doença do trato urinário inferior dos felinos: abordagem sobre cistite idiopática e urolítiase em gatos. Enciclopédia Biosfera. 15(27), 134-148.

Ázira, P.C. (2016). Tratamento das urolitíases em cães e gatos: abordagens não cirúrgicas. Enciclopédia Biosfera. 13(23), 1314-1335.

Bartges, J.W. (2016). Feline calcium oxalate urolithiasis. Journal Of Feline Medicine And Surgery, 18(9), $712-722$.

Barwaldt, E. T., Bierhals, E. S., de Lima, C. M., da Silva, A. B., \& Ferraz, A. (2021). Urolitíase em filhote canino: Relato de caso. Research, Society and Development, $10(1)$.

Carvalho, C. F. (2014). Ultrassonografia em pequenos animais. (2a ed): Roca, 468

Del-Angel-Caraza J., Mendoza-López C.I., Aguiñaga-Negrete E. \& Quijano-Hernández I.A. (2020). El gato com urolitiasis de oxalato de calcio o estruvita. Remevet. v. 4, n. 1, p.5-10.

Ettinger, S. J., Feldman, E. C. \& Cote, E. (2017). Textbook of Veterinary Internal Medicine-eBook. Elsevier Health Sciences.

Gomes, V. R., Ariza, P.C., Borges, N. C. Schulz, F. J. \& Fioravanti, M. C. S. (2018). Risk factors associated with feline urolithiasis. Veterinary Research Communications. 42(1), 87-94.

Grauer, G. (2015). Manifestações clínicas dos distúrbios urinários; Urolítiase canina. In A. W. Nelson \& C. G. Couto (Eds.), Medicina Interna de Pequenos Animais. Elsevier Editora.

Grauer, G. F. (2005). Canine glomerulonephritis: new thoughts on proteinuria and treatment. Journal of Small Animal Practice, 46(10):469-478.

Houston, D. M., Vanstone, N. P., Moore, A. E. P., Weese, H. E. \& Weese, J. S. (2016). Evaluation of 21426 feline bladder urolith submissions to the Canadian Veterinary Urolith Centre (1998-2014). The Canadian Veterinary Journal, Canada, 57(2),196-201.

Lekcharoensuk, C., Osborne, C. A., \& Lulich, J. P. (2001). Epidemiologic study of risk factors for lower urinary tract diseases in cats. Journal of the American Veterinary Medical Association, 218(9), 1429-1435.

Little, S. E. (2016). Trato urinário inferior. In O Gato - Medicina Interna (pp. 944 - 975). Rocca.

Lulich, J. P., Osborne, C. A., \& Albasan, H. (2011). Canine and feline urolithiasis: diagnosis, treatment, and prevention. In Nephrology and urology of small animals (pp. 687-706). Wiley.

Mendoza-López, C.I., Del-Angel-Caraza, J., Aké-Chiñas, M.A., Quijano-Hernández, I. \& Barbosa-Mireles, M. (2019). Epidemiology of feline urolithiasis in Mexico (2006-2017). Journal of Feline Medicine and Surgery Open Reports. 5(2), 1-6.

P Souza, L. D. P. De.; Rocha, M. M.; Ferrando, J. T.; Moraes, L. A. M. De; Ramos, A. J. R. T.; Madril, A. B.; Bilhalva, M. A; Amaral, A. Do; Soares, R. C.; Boff, G. A.; Miranda, J. V.; Maggi, V. B..; Cavalcante, G. A. De O.; Grecco, F. B. (2021). The Role Of Urolithiasis In Urethral Obstruction In Cats: A Literature Review. Research, Society And Development. 10 (8).

Rick, G.I., Conrad, M. L. H., Vargas, R. M., Machado, R. Z., Lang, P. C., Serafini, G.M. C. \& Bones, V. C. (2017). Urolitíase em cães e gatos. Pubvet, Londrina, 11(7), $705-714$.

Silva, P. K. G., Silveira, E. B., Lima, D. J.S. \& Kobayashi, Y. T. S. (2020). Diagnóstico e terapia de urolitíase em um felino: relato de caso. Pubvet, 14(3), 1-8.

Stevenson, A. E. (2002). The incidence of urolithiasis in cats and dogs, and the influence of dietin formation and prevention of recurrence (Doctoral dissertation, University of London, University College London (United Kingdom)).

Waki, M. F. \& Kogika, M. M. (2015). Urolitíase Em Cães E Gatos. In: Jericó, M. M., Neto, J. P. A.\& Kogika, M. M. Tratado de Medicina Interna de Cães e Gatos. Roca, Cap.165, p. 1462-1482.

Yin, R. K. (2015). O estudo de caso. Bookman. 